\title{
EVOLUTION MIKRO - MICRO-DOSING IN THE HIGH-PRESSURE RANGE THANKS TO INNOVATIVE DRIVE TECHNOLOGY
}

\author{
Bernd Freissler \\ ProMinent GmbH, Im Schuhmachergewann 5-11, 69123 Heidelberg \\ Tel.: +49621 842507; E-mail freissler.bernd@prominent.com
}

\begin{abstract}
Oscillating positive displacement pumps are used in many industrial sectors. Mechanical stroke generators / drives such as crank drive, spring-cam drive etc have reached a high technological level, but to which are set mechanical limits. Especially in the smallest dosing range $<1 \mathrm{l} / \mathrm{h}$ at a pressure range from $100 \ldots 400$ bar considerable optimisation is still required with regard to precision and continuity / control range of the dosing flow. In order to expand the current application possibilities for the diaphragm metering pump technology, it is necessary to use new drive systems such as linear motor technology. A linear motor is an electric drive unit which transmits the oscillating delivery movement of the dosing pumps directly to the displacer (hydraulic piston, diaphragm, etc.) without any mechanics, so that highly dynamic movements can be carried out with maximum precision, an individual kinematic profile and a control range of 1:200. The examples of gas odorization and filling processes show how the linear motor drive can be used to technologically solve and even optimize the customer's process requirements. The linear motor pump can realize an integrated 3-parameter control and is therefore suitable for almost any kinematically solvable dosing task.
\end{abstract}

Keywords: metering pump with linear-motor drive, integrated 3-parameter-control

\section{INTRODUCTION}

Mechanical stroke generators have a wide range of applications across all branches of industry. From simple lifting bow saws to windscreen wiper drives to tool drives (shapers) for machining or as piston compressors and pumps. One of the most important applications are oscillating positive displacement pumps.

In the following article, I would like to discuss the functional groups of oscillating positive displacement pumps with adjustable stroke length and ask the possibly most important question "Which drive/pump system is capable of optimally adapting to a dosing task?"

\section{BASICS}

\subsection{Definition and operation of dosing pumps}

The influence of fluctuations in process parameters on the flow rate is lowest in the oscillating positive displacement pump, as Figure 1 and 2 show.

Most dosing pumps have a linear dependence between the manipulated variables (stroke length $\mathrm{h}$ and stroke frequency $\mathrm{n}$ ) and the dosing current Q. They can therefore be easily integrated into automated processes.
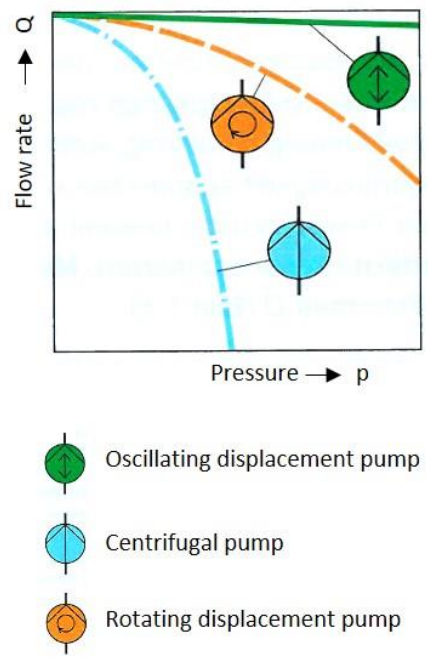

Figure 1: Dependence of the flow rate $Q$ on the discharge pressure $p$ of the dosed fluid 

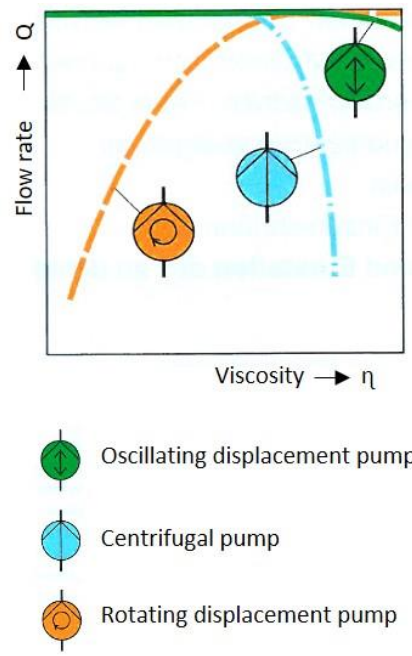

Figure 2: Dependence of the flow rate $Q$ on the viscosity $\eta$ of the dosed fluid

Therefore, and also because of its principle of action, it is particularly suitable for dosing tasks supplemented by some additional functions:

- It delivers and increases the pressure level like any other pump.

- It measures by repeatedly displacing a given stroke volume (measuring cup principle).

- Its flow rate can be adjusted by continuously changing the stroke length, stroke volume or stroke frequency.

Apart from some special designs, dosing pumps are equipped with automatic valves which are controlled by the pressure change in the pump working chamber. Figure $\mathbf{3}$ explains the operating principle of the classic dosing pump.

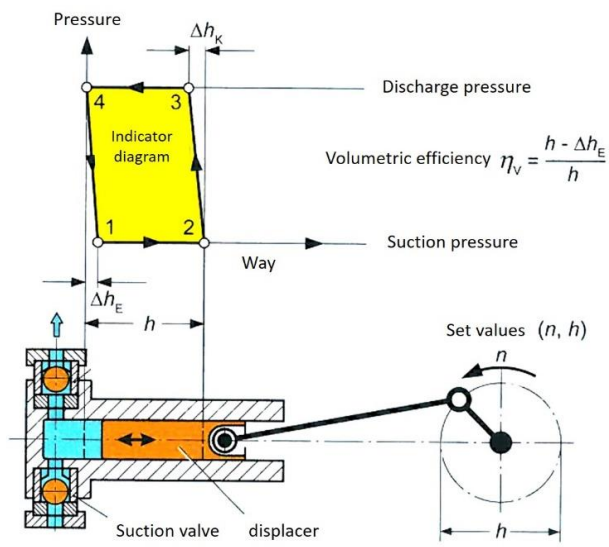

Figure 3: Function of the dosing pump

\subsection{Mechanical drive units}

The kinematic principles of the best-known stroke-adjustable dosing pump drives, such as straight slide crank drive, rotary crank or springcam drive, etc. are shown in Figure 14.

There are basically two parameters available for setting the flow rate in these drives.

- Stroke length: The mechanical setting of $0-100 \%$ is possible by means of a handwheel or an electric stroke length setting or control motor.

- Stroke frequency: The flow rate is set with the number of strokes per time unit (strokes/min), e.g. by means of a frequency converter control of the drive motor.

A special form of the oscillating positive displacement pump is the electromagnetic linear drive, as Figure 4 shows. The solenoid generates the discharge stroke with its energy field, whereas the suction stroke is executed with a mechanical force such as a return spring. This is a widely used system in the low power range up to 100 watts.

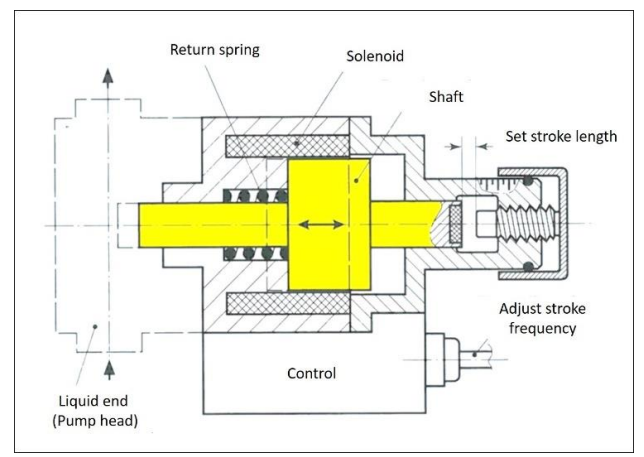

Figure 3: Function of the dosing pump

\subsection{Liquid ends}

The dosing units/liquid ends are essentially differentiated according to the displacement type, whereby only the diaphragm systems are dealt with here (Figure 5b and 5c). 


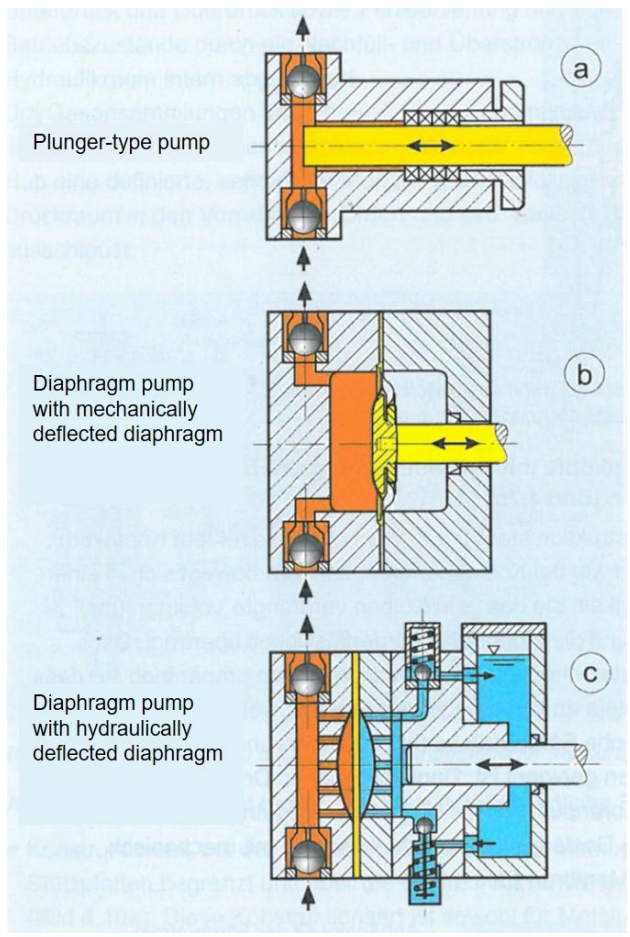

Figure 5: Different types of liquid ends

\section{Mechanically deflected diaphragm version}

The mechanically deflected diaphragm (Figure 5b) is typically used in the low-pressure range up to 20 bar (occasionally up to 25 bar).

Advantages:

- Hermetically tight

- Simple design (cost-effective)

- Accuracy at $\pm 2 \%$, with a stroke length adjustment range of 1:4

Disadvantages:

- Not suitable for medium and high pressures

- No integrated overpressure protection in the liquid end

\section{Hydraulically deflected diaphragm version}

The hydraulically deflected diaphragm (Figure 5c) is very frequently used in the process industry (oil, gas, chemicals, etc.) in the pressure range up to 400 bar (occasionally up to 3,000 bar).

Advantages:

- Hermetically tight

- Accuracy at $\pm 1 \%$, with a stroke length adjustment range of 1:10
- In the liquid end integrated hydraulic pressure relief valve as overpressure protection.

Disadvantages:

- Technically complex solution (cost-intensive)

\subsection{Characteristic transfer features}

The oscillating movement of the displacer results in a digital/pulsating transfer characteristic (Figure 6) with a pressure-stable curve (Figure 7) and a simple linear dependence (Figure 8) of the dosing quantities on the setting parameters stroke length and stroke frequency.

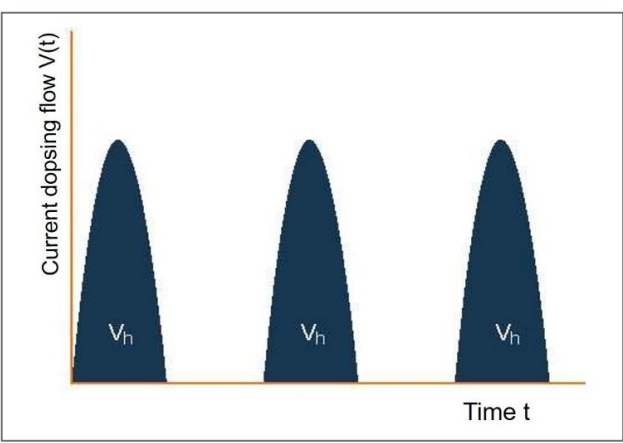

Figure 6: Pulsating characteristic of transfer

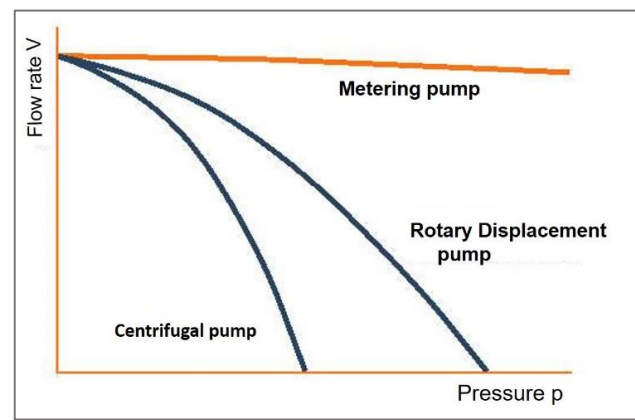

Figure 7: Pressure-resistant curve

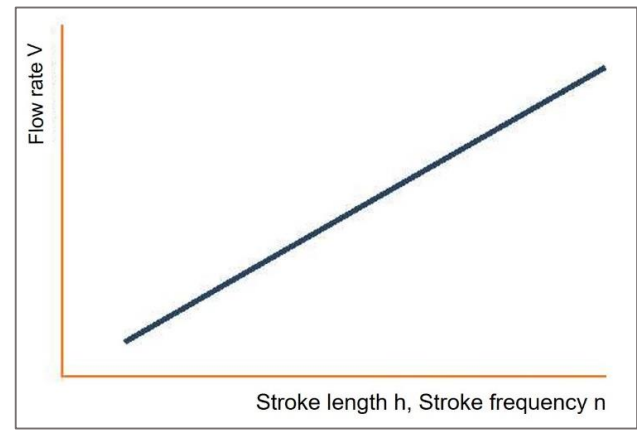

Figure 8: Simple linear dependence 
When it comes to hydraulic efficiency, oscillating displacement / dosing pumps with typical values of $75-95 \%$ are the most efficient system among the feed pumps.

\section{CHALLENGE - OVERCOMING MECHANICAL LIMITS OF DRIVE}

\subsection{The process step dosing}

Many process engineering procedures require precise and reliable dosing of material components to ensure that the end product produced meets the requested quality requirements. As more and more production processes have been fully automated in recent decades (Figure 9), dosing equipment is needed that can be integrated into the workflow of an overall process as simply and efficiently as possible. It has been shown that this is most easily achieved if the substances to be dosed are flowable, because this not only simplifies the process sequence, but also enables high dosing accuracies to be realized. Solids are therefore often prepared in fine-grained form and suspended or dissolved in a suitable carrier liquid. Each dosing process consists of three individual steps:

- Transfer (convey)

- Measure and

- Adjust the dosed flow rate Q

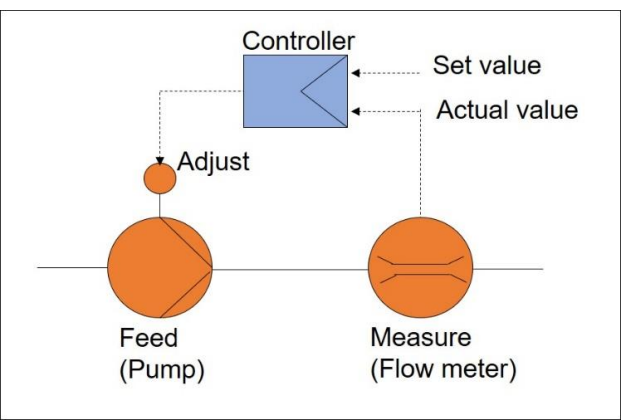

Figure 9: Dosing in the control circuit

\subsection{Dosing parameters}

Especially with regard to the drive technology of motor-driven dosing pumps, it can be stated that the kinematic principles of dosing movement (path-time profile, etc.) have virtually not changed in recent decades (see Figure 10).

Although there are already some systems such as vector-controlled frequency converter pumps or controlled solenoid drives that modify the path-time diagram, these are still far from a true free definition of the complete motion profile.

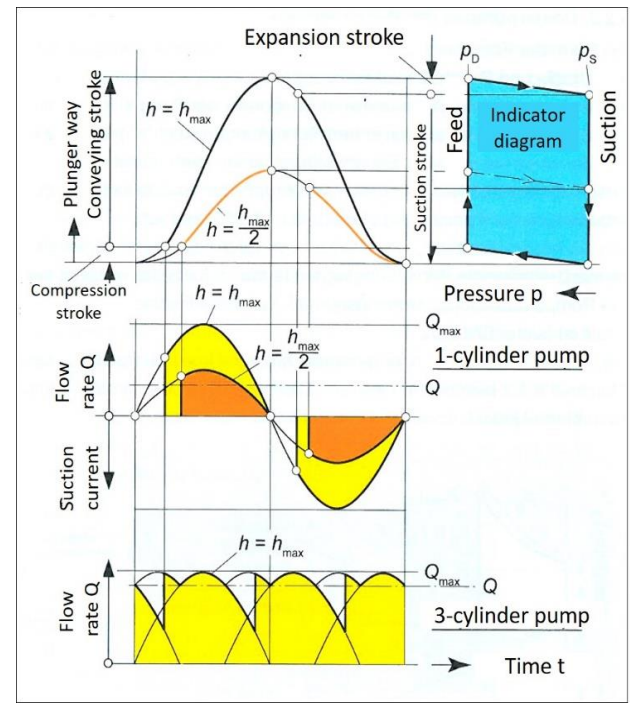

Figure 10: Flow rate pulsation $\mathrm{Q}(\mathrm{t})$ of a dosing pump with harmonic kinematics and volumetric efficiency $n_{\mathrm{y}}=0.9$

The process of dosing still essentially includes the classic 1 or 2 parameter control:

- Stroke volume/stroke length control

- Stroke frequency/frequency converter for controlling the motor speed

\section{Parameter 1 - stroke volume / stroke length control}

Classically, the stroke length is set between $0-100 \%$ (either mechanically or electrically supported). This process is typically carried out with a separate device for mechanical stroke length adjustment (adjusting eccentric, remote cam drive, etc.) or by means of an electric rotary drive. In practice, a typical control range of up to 1:10 is implemented here.

\section{Parameter 2 - stroke frequency / frequency converter for drive motor}

The speed of the electric drive motor in the control range $1: 3$ to $1: 5$ is usually controlled by a frequency converter. 


\section{Parameter 1 and 2 in combination}

In order to realize a larger overall control range, it is necessary to use both parameters in combination. In practice, control ranges from $1: 20$ to $1: 30$ can be reliably realized (theoretically 1:50). The control ranges of the individual parameters are either connected in series (via process control system) or are linked to two independent control circuits.

\subsection{Future requirements on dosing technology}

Ideally, the operator would like to have a dosing solution for his application that is individually adapted to his needs.

In the best case, this means a 2-parameter control with a variable motion profile (parameter 3), which is exactly matched to the requirements during the injection of the pump into the process (discharge stroke) and, considered separately, also to the design of the suction stroke.

The properties of the liquids to be dosed, such as viscosity, chemical resistance, etc. must be taken into account, as must the hydraulic characteristics of the dosing stroke. These include, for example, minimising the spraying behaviour at the nozzle and preventing postdripping in filling processes.

\section{SOLUTION WITH LINEAR MOTOR TECHNOLOGY}

\subsection{Basics}

The actual dosing movement required is the oscillating dynamic stroke in both directions. Therefore, a drive unit that executes a directly controlled/regulated oscillating movement for discharge and suction stroke without any movement and force deflection would be optimal. The linear motor fulfils exactly this requirement. The supplied electrical energy is converted directly into an oscillating movement. By this construction the complete drive unit contains only one moving part (Figure 11).

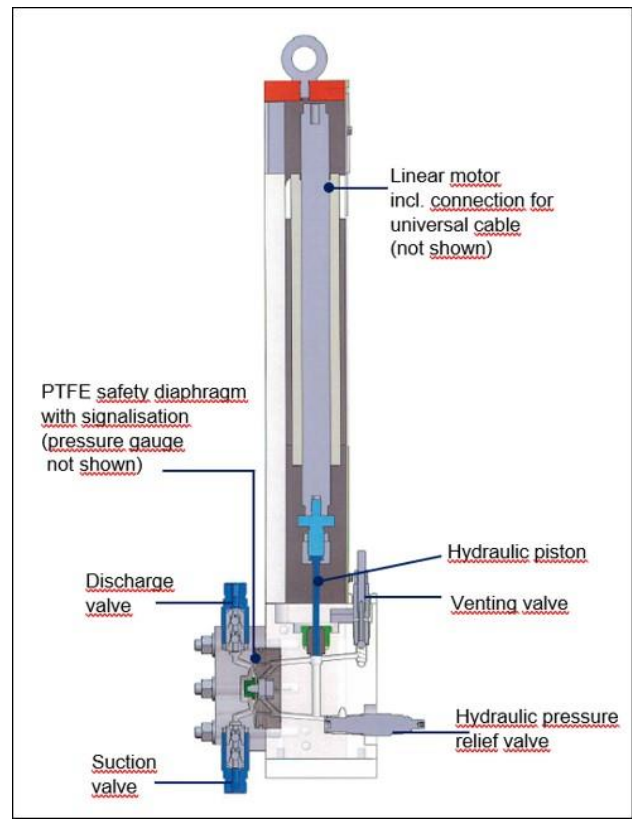

Figure 11: Structure of the linear motor pump

The structure and function of the linear motor are shown in Figure 12. The stator length with the coil and solenoid pairs is proportional to the force. The excess length of the solenoid package generates a constant force over the entire process range.

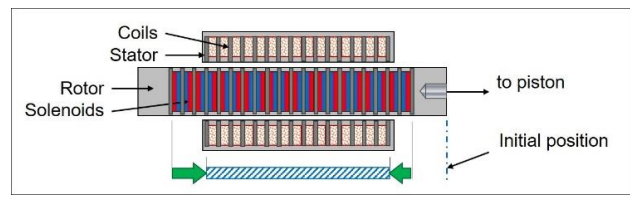

Figure 12: Structure and function of the linear motor

In comparison, the movement of the electric rotary motor is reduced from the motor speed (e.g. $1400 \mathrm{rpm}$ ) to the required stroke frequency for dosing pumps (e.g. 1:10 which corresponds to 140 strokes/min.) by means of a gear reduction. This is then converted into a usable oscillating movement via a crank drive with adjustable eccentric. Three changes are therefore ultimately necessary in order to obtain the actually usable, adjustable oscillating movement type. Figure 13 shows the replacement of these components in linear motor technology. 


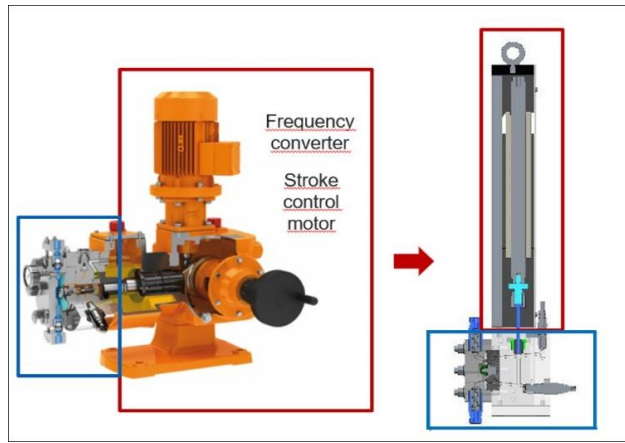

Figure 13: Comparison of mechanical stroke generator and linear motor

The advantages of the linear motor are obvious:

- The gearless design allows operation without deflection. This reduces components, making the design relatively wear-resistant. Costs and installation space can also be saved.

- Due to the high dynamics (velocity/ acceleration) inadequacies in the hydraulics, e.g. in the valves, can be actively compensated.

- The "stepper motor characteristic" with integrated path detection is well suited for the control of dosing profiles (see Figure 15 17).

- Unlike solenoids, the force is proportional to the current and can be applied in both directions.

Disadvantages:

- Low drive power, currently only up to $1 \mathrm{~kW}$ is possible.

- A drive control is always required for operation.

- Realisation

During the practical implementation, it should of course be noted that the combination of

- long stroke,

- highly dynamic motion and speed sequences and

- relatively low rod force

is best used in combination with a hydraulic diaphragm dosing unit (Figure 5c). Despite the low rod force of the linear motor, the hydraulic piston/force ratio makes it possible to achieve high conveying pressures such as 400 bar.

Due to the large stroke length, a useful stroke volume is possible despite the small piston diameter. Furthermore, the large stroke length also enables very precise positioning of the piston, which is reflected in a very high accuracy of the dosing flow.

\subsection{Parameter control $2+1$}

A linear motor is usually combined with its own individual control unit, so that the typical operating modes for 1 or 2 parameter control can be selected variably. These are e.g.

- Manual / permanent operation

- Contact activation

- Analogue control

- Fieldbus control (e.g. ProfiNet)

It is also possible to program a fixed or dynamic dosing profile for the discharge and (separately) for the suction stroke as a third parameter.

This means that the pump controller is on the one hand able to combine the stroke length and stroke frequency parameters optimally (e.g. $60 \%$ stroke length with $40 \%$ stroke frequency) and on the other hand at the same time to change/adjust the path-time profile according to requirements.

The linear pump can implement an integrated 3-parameter control and is therefore suitable for almost any kinematically solvable dosing task.

The best way to demonstrate the advantages of linear motor technology is to use the dosing profiles. Figure 14 shows the kinematics of the classic crank drive/spring cam drive. 


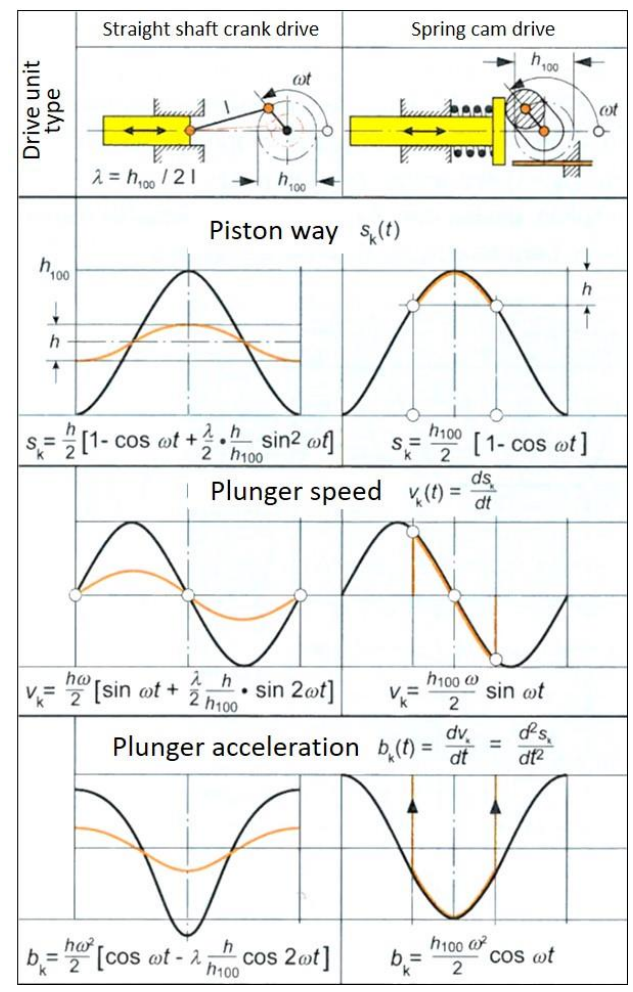

Figure 14: Kinematics of the straight shaft crank and spring cam drive at full stroke and reduced stroke length

In comparison, Figures $\mathbf{1 5}$ to $\mathbf{1 7}$ show the flexibility of the linear motor on the basis of different speed-time profiles of single-head pumps. These are classified in the following chapter by means of application requirements and application examples.

\section{APPLICATION EXAMPLES}

\subsection{Continuous dosing}

\section{Dosing task}

Optimum mixing by means of almost continuous dosing

\section{Application:}

For gas odorization it is requested that the dosing quantity of odorant (e.g. mercaptans) is introduced as evenly as possible into the gas flow. This means that the suction stroke takes place in short time intervals $(<0.5 \mathrm{sec}$.) and the pressure stroke in as long time intervals as possible (up to
30 sec.). A linear motor drive can carry out the suction and discharge sides completely independently of each other without any problems and thus carry out the corresponding dosing profiles.

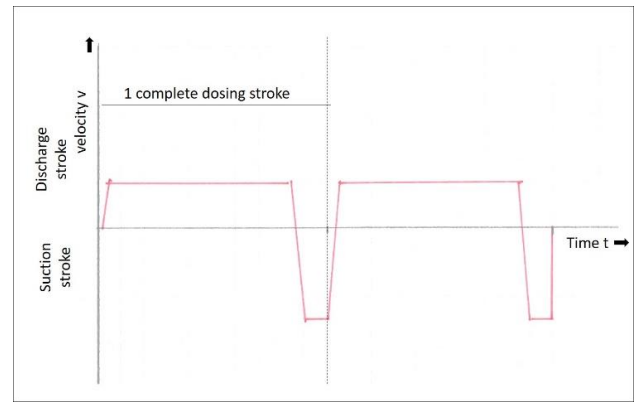

Figure 15: Linear motor dosing pump Evo mikro nearly continuous dosing with minimum dosing pause

\subsection{Dosing of high viscous fluids}

\section{Dosing task}

Dosing of highly viscous fluids e.g. polymers with $1,000 \mathrm{mPas}$ in water treatment

\section{Application:}

In order to achieve optimum dosing of highly viscous media, it is important that the liquid is sucked in during the suction stroke with the displacer moving as evenly and relatively slowly as possible. Furthermore, the pressure stroke must be performed much faster in order to reduce the backflow leakage at the valve. A linear motor drive can easily execute the suction and discharge sides completely independently of each other and thus execute the corresponding dosing profiles.

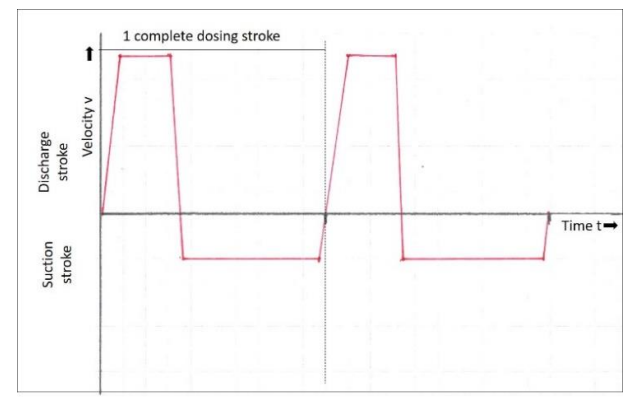

Figure 16: Linear motor dosing pump Evo mikro dosing of high viscous fluids, optimized with long suction stroke 


\subsection{Dosing in the filling process}

\section{Dosing task}

Dosing of additives in measuring cups with high accuracy, variable cycle times and absolutely drip-free

\section{Application:}

In the laboratory, individual test samples of various fluids are required for various processes, i.e. for every possible combination of process and fluid it is necessary to adapt the filling process (dosing profile). Figure 17 shows the filling of a viscous additive.

1. Phase - Pressure stroke

Start at medium speed to open all valves quickly and set the liquid in motion.

2. Phase 2 - Constant speed

To continuously fill the fluid into the measuring cup.

3. Phase 3 - Gentle speed reduction To prevent dripping

4. Phase 4 - Suction

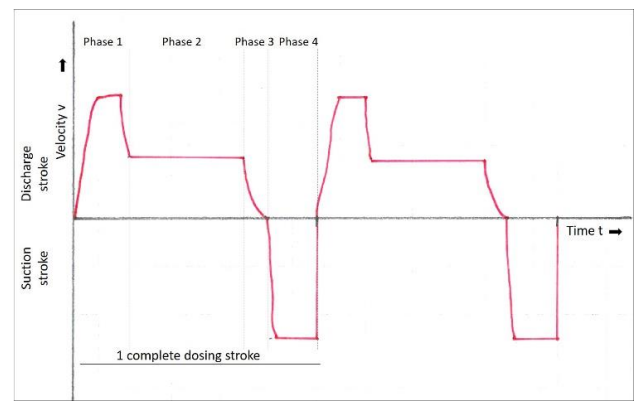

Figure 17: Linear motor dosing pump Evo mikro filling process with nozzle due to individual and optimized speed profile with drop-free effect

\section{CONCLUSION}

Returning to the question: "Which drive/pump system is capable of optimally adapting to a dosing task?", we have found a new answer. By using a highly dynamic and precise linear motor as drive for oscillating positive displacement pumps, you have all degrees of freedom to adapt the pump kinematically to the dosing task.

Such an individual/application-optimised kinematic profile is particularly important in the field of small and very small quantity dosing in combination with medium and high pressures.

Consequently, the next question is: "What do the kinematic dosing profiles for the most varied fluids (high viscosity, solvents, etc.) and the various processes (filling quantity, continuity, dosing profile, etc.) have to look like in order to maintain an optimum?

With linear motor technology, we will systematically determine the different profiles and thus contribute to plant optimization.

\section{REFERENCES}

[1] Dulger V (Hrsg.), 2020 Richtig Dosieren, Vogel Business Media, Würzburg, Germany

[2] Fritsch H (1989) Die Bibliothek der Technik Bd. 50, Dosierpumpen - Grundlagen, Konstruktionen, Anwendung, verlag moderne industrie, Landsberg/Lech, Germany

[3] Vetter G (Hrsg.), 1994 Handbuch Dosieren, Vulkan Verlag, Essen, Germany 